\title{
Challenges in Retrorectal Cysts: Is Preoperative Diagnosis Essential?
}

\author{
Kursat Rahmi Serin ${ }^{1}$, Onder Karabay ${ }^{2}$, Nadir Adnan $\mathrm{Hacım}^{3}$ and Mustafa Cem Terzi ${ }^{2}$ \\ ${ }^{1}$ Istanbul Faculty of Medicine, Istanbul University, Istanbul, Turkey \\ ${ }^{2}$ Department of General Surgery, Surp Pirgiç Armenian Hospital, Istanbul, Turkey \\ ${ }^{3}$ Department of General Surgery, Bagcilar Training and Research Hospital, Istanbul, Turkey
}

\begin{abstract}
Developmental cysts are a subgroup of perirectal cysts seen extremely rarely. They have no symptoms in $50 \%$ of cases, and mostly occur among middle-aged women. The ratio of male to female is approximately $1 / 3$. In the differential diagnosis, malignancies and pilonidal cysts are common. Although they usually occur as benign lesions, $30 \%$ of cases are reported as malignant in literature. Preoperative diagnosis is quite challenging, but may be essential for appropriate treatment. The main treatment is surgical removal of the entire cyst for the prevention of complications and the potential for malignancy. In addition to the trans-sacral or perineal approach, anterior laparoscopy can be performed as an optional surgical technique.

Herein, we report two consecutive patients with two different developmental cyst pathologies. A 17-year girl, who had no specific symptoms for a retrorectal giant cyst, was admitted to this clinic because of pilonidal cyst disease. A giant retrorectal cyst was diagnosed through pelvic MRI, which was performed upon her reporting a mild fullness feeling. The other cyst was diagnosed in a 30-year woman who had mild gastrointestinal symptoms that could not be related to the cyst. Both underwent surgery via the trans-sacral approach. The cysts were completely removed without abdominal intervention.
\end{abstract}

Key Words: Epidermoi cyst, Pilonidal cyst, Tail-gut cyst, Retrorectal Space.

How to cite this article: Serin KR, Karabay O, Hacım NA, Terzi MC. Challenges in Retrorectal Cysts: Is Preoperative Diagnosis Essential?.J Coll Physicians Surg Pak 2020; 30(04):429-432. DOI: https://doi.org/10.29271/jcpsp.2020.04.429.

\section{INTRODUCTION}

In the adult population, developmental cysts are the most common cystic lesions located in the retrorectal pelvic area. This space is bound by the peritoneal reflection and the rectosacral fascia. These cysts are classified under 4 subgroups: tail-gut (rectal duplication) cysts, epidermoid cysts, dermoid cysts, and teratomas. They are extremely rare, and more common in females. ${ }^{1-3}$ They are usually asymptomatic and mostly diagnosed incidentally in radiologic tests done for unrelated diseases. ${ }^{2.4}$ They present as slow-growing, painless, and well-demarcated cysts, arising from dermal or subcutaneous epidermal tissue. ${ }^{1-3}$ Radiologic differential diagnosis is quite challenging because findings are similar in most kinds of cysts. Preoperative biopsy is not feasible in most cases. It is not diagnostic/adequate because of the thin cyst wall and hypocellular cyst liquid. Enforced biopsy could cause major complications such as rectal injury and abscess. ${ }^{5}$ Removal of the cyst in its entirety is the main goal of treatment. The preferred surgical approach for appropriate candidates, especially for small cysts, is the trans-sacral approach. ${ }^{1}$

Correspondence to: Dr. Nadir Adnan Hacım, Department of General Surgery, Bagcilar Training and Research Hospital, Istanbul, Turkey

E-mail: adnanhcm@hotmail.com

Received: March 28, 2019; Revised: July 30, 2019;

Accepted: August 22, 2019

DOI: https://doi.org/10.29271/jcpsp.2020.04.429
Herein, we report two consecutive patients who underwent surgery for giant pelvic cysts that were pathologically diagnosed as two different types of developmental cysts. Both patients underwent surgery via the trans-sacral approach to avoid abdominal complications.

Case 1: A 17-year girl was admitted to another clinic due to leakage of seropurulent fluid and pain in the intergluteal cleft for 3 months. Pilonidal sinus was diagnosed and surgical removal was recommended. When she came to this clinic for surgery, she stated that she experienced a vague sensation of fulness in the left gluteal region on sitting. She had three history of constipation or pain. She had 3 fistula orifices at the intergluteal area with leakage of serous liquid. On digital rectal examination, a smooth, cystic lesion projecting from the posterior wall of the rectum without any mucosal finding, was palpated.

Contrast-enhanced pelvic magnetic resonance imaging (MRI) revealed a perirectal $13 \times 7 \mathrm{~cm}$ cystic mass with a very thin and pressurised wall seen in the retrorectal area. No thickening of the wall or nodularity was seen. The cyst arose from the left part of the presacral region and reached the gluteal subcutaneous tissue (Figure 1a). It was very close to the posterior wall of the rectum. In addition, there was a $28 \times 7 \mathrm{~mm}$ fistulous tract with a small cyst at the inferior side of the coccyx (Figure 1b). The coexistence of pilonidal disease with the perirectal cyst was diagnosed and surgical removal of both throughthetrans-sacral approach was planned.

The epidermal and pilonidal cysts were excised together in a semibent face-down (jackknife) position; the incision started from the subcutaneous tissue and continued to the anal sphincter region, 
reaching the mesorectum and rectal wall. The cyst was completely removed with transanal digital guidance to prevent rectal injury (Figure 2). There was no communication between the epidermoid and pilonidal cysts. A suction drain was placed and the incision was closed primarily. She was discharged on the 4th day after surgery with no complications.

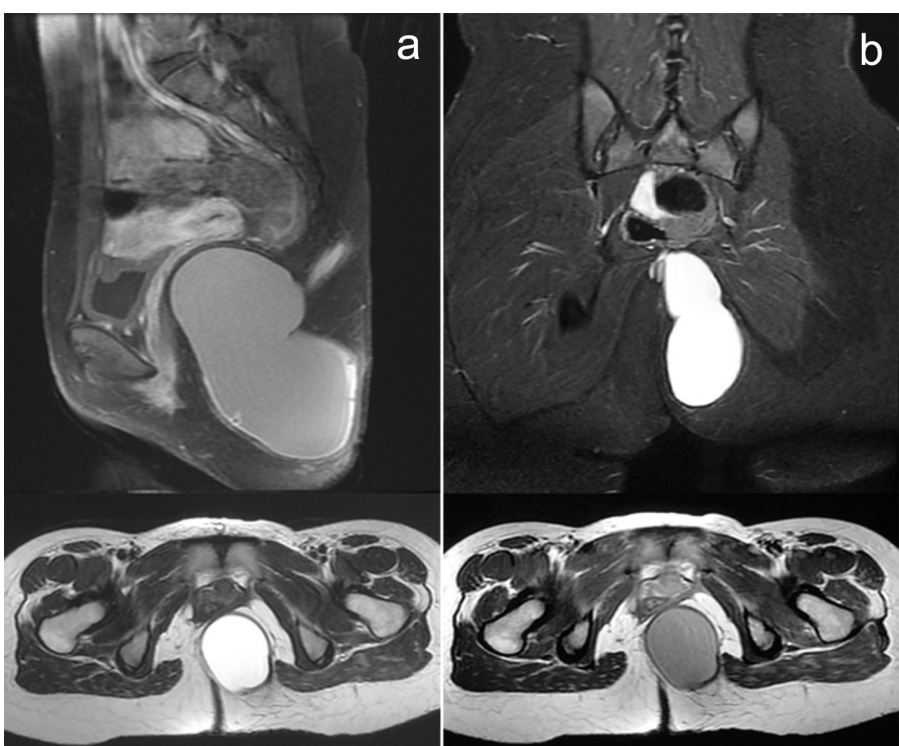

Figure 1: Pelvic MRI: (a) Giant, retrorectal epidermoid cyst, (b) pilonidal sinus. Cyst in the left gluteus, rectum displaced anteriorly.

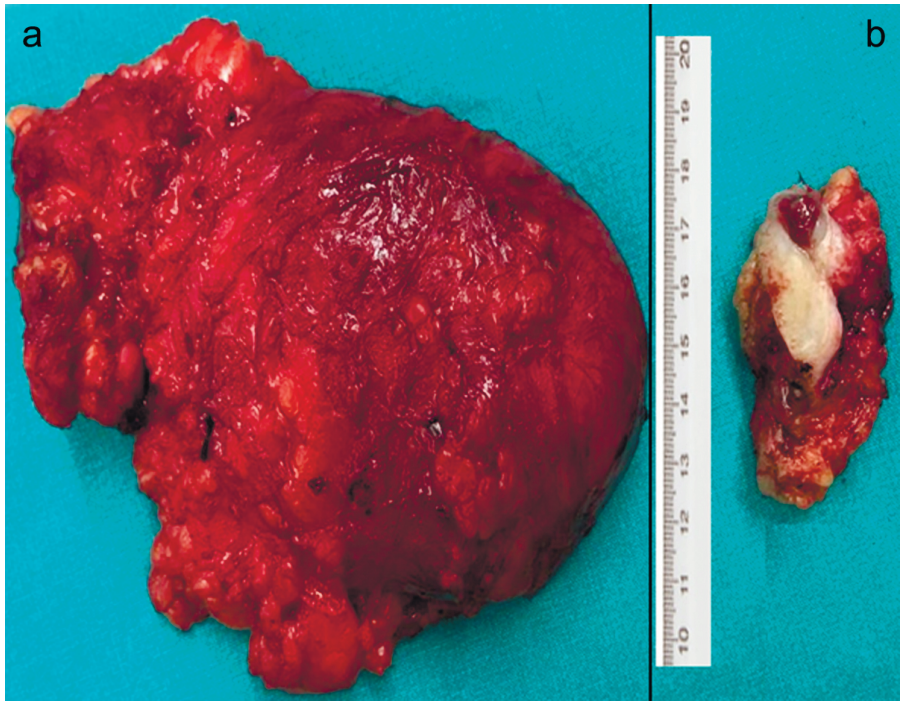

Figure 2: (a) Epidermoid cyst, (b) Pilonidal cyst.

In the pathological examination, the wall of the cyst was lined by stratified squamous epithelium and filled with keratinised material. Inflammatory cell infiltration with foreign body-like giant cells was observed. The cyst was diagnosed as an epidermoid cystic tumor that was benign in nature (Figure 3). The pilonidal cyst also had stratified squamous epithelium, and a sinus structure-fistulous tractincluding hair with inflammatory cell infiltration.

Case 2: A 30-year woman was admitted to another clinic due to constipation and pelvic pain upon sitting. There were no clinical findings in her examination. The physicians performed contrastenhanced pelvic MRI and a perirectal $15 \times 8 \mathrm{~cm}$ cystic mass with a thickened and pressurised wall was seen. The cyst wall was approximately $4 \mathrm{~mm}$ thick withoutany irregular-soft tissuecompo- nent, and the cyst was filled with a water-like liquid. The thickened cyst wall showed homogenous contrast enhancement without any nodularity. This hourglass-shaped cyst arose from the left part of the presacral region, consisting of two parts with an interconnection (Figure 4). The cyst was located posterior to the rectum, It compressed and displaced the rectum anteriorly with no findings of invasion.

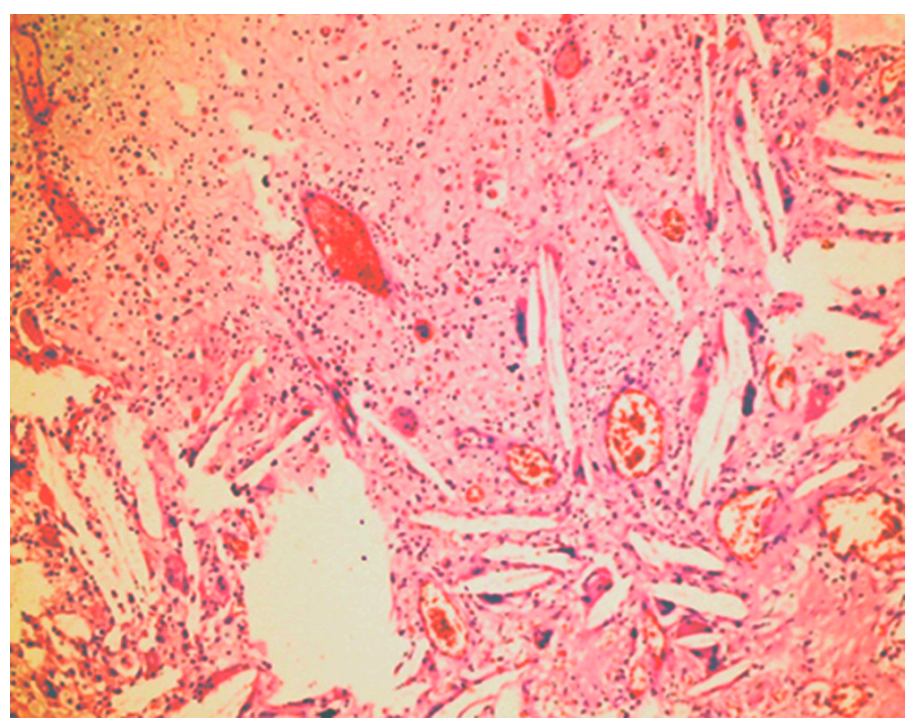

Figure 3: Epidermoid cyst, lined with stratified squamous epithelium and keratin with giantcells.
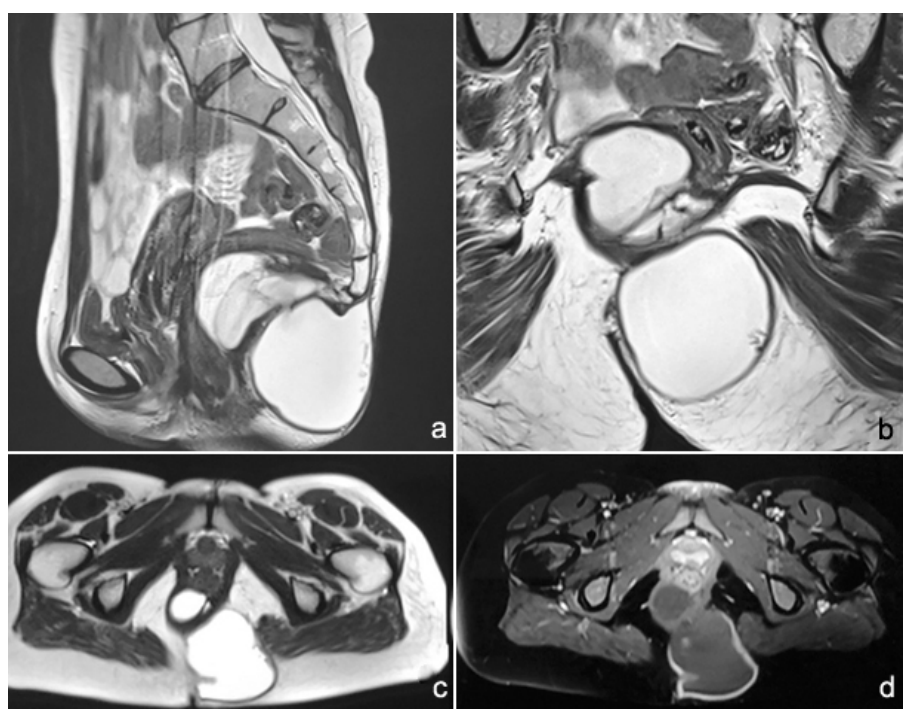

Figure 4: (a) Hourglass-shaped, interconnected two parts of the cyst. $(a, b, c)$ Cyst filled with clear, water-like liquid. (a,c,d) Rectum displaced anteriorly.

The cyst was excised in the jackknife position; the incision started from the left gluteal subcutaneous tissue, reaching the mesorectum and rectum wall. There was no rectal wall invasion and complete excision of the cyst was performed. Transanal digital guidance was also used in this case. A suction drain was placed and the incision was closed primarily. She was discharged on the 3rd day after surgery with no complications.

Histopathologically, the cyst wall was lined by stratified squamous epithelium with adnexae. Inflammatory cell infiltration with foreign body-like giantcells, patchycalcification, and fibro-hyalinisation were seen. The cyst was diagnosed as a tail-gut cyst with no findings of malignancy. 


\section{DISCUSSION}

Perirectal/retroperitoneal pelvic cystic masses are extremely rare, and they are divided into 5 categories: congenital (developmental), inflammatory, neurogenic, osseous, and others. ${ }^{1}$ Developmental cysts comprise epidermoid, dermoid, tail-gut (hamartoma) cysts, and teratomas. They originate from an ectodermal remnant that is misplaced during embryogenesis. ${ }^{1}$ More than $50 \%$ of such cysts are asymptomatic and are usually diagnosed incidentally. Generally, symptoms develop based on the mass effect such as rectal fullness, painful defecation, constipation and dysuria, and fistula formation. An abscess may be seen in complicated cases. ${ }^{2,4}$ In The first case, the patient had only fullness feeling upon sitting and this situation was not paid attention to previously. The second patient was admitted to a clinic due to symptoms of constipation and pain.

Digital rectal examination and inspection is a low cost, simple, and routine way to detect rectal-perirectal pathologies. More than $75 \%$ of perirectal massescan be palpated through digital examination, and an inspection could lead to the diagnosis. ${ }^{5}$ In The first case, where the patient reported rectal fullness feeling, the cyst was palpated through digital examination without any mucosal defect. She had pilonidal cystic disease with a fistulous tract that could have easily caused misdiagnosis by masking these mild symptoms/findings. ${ }^{6,7}$

Axial radiologic imaging methods [computed tomography (CT) or MRI] for diagnosis are better than endoscopy, especially in cases detected in intact mucosa. ${ }^{3,5,8,9}$ Pelvic MRI is usually preferred over $\mathrm{CT}$ in retroperitoneal, and perirectal pathologies ${ }^{7}$ because the pelvic area is immobile and MRI scans provide better information, especially about the form (cystic or solitary), nature, malignant potential, and the proximity or invasion of neighboring organs. ${ }^{3,5}$ In both cases contrast-enhanced MRI. Was used: Both cysts were arising from the left side of the presacral region and reached the gluteal subcutaneous tissue (Figure 1-4). Both were huge, retrorectal, cystic masses, filled with clear water-like liquid and had pressurised walls. In the second case, there were thin septae inside the cyst and a thickened wall was seen without irregularity, with no suspicious findings for malignancy as per homogenous contrastenhancement. Regarding the proximitiestoriskyorgans/vasculature as described in MRI, both cysts were very close to the posterior wall of the rectum with no invasion. In the first case, there was a $28 \times 7 \mathrm{~mm}$ fistulous tract with a small pilonidal cyst at the tip of the coccyx (Figure 1b). MRI guidance provided for better decisions regarding access, informing us to take care to avoid rectal injury.

Endorectal ultrasound (EUS) could give information about the form and invasion or proximity to organs, but is not better than an up-to-date MRI. ${ }^{9}$ EUS could also provide the opportunity for biopsy for a definitive diagnosis, which is not preferred for inoperable, cystic lesions, especially in thin-walled, possibly benign cysts. ${ }^{3,5}$ Enforced biopsy could be risky for rectal injury or infection, and it could lead to spread into the abdominal cavity in the case of malignancy. $3,5,9$

Most cases are benign and although epidermoid cysts rarely become malignant, early surgical resection is the recommended treatment. ${ }^{3,9}$ Radiologic findings are also important for mapping the surgery. Transabdominal, trans-sacral, trans-rectal or combined methods could be used for resection. ${ }^{1,5}$ The posterior approach (trans-rectal, trans-sacral) is usually the preferred access to prevent intraabdominal complications for appropriate candidates. ${ }^{5}$ The anterior laparoscopic approach can be successfully performed. Laparoscopic resection has recently been reported as a valid alternative to other abdominal procedures, with reduction of surgical trauma. ${ }^{6}$

MRI scans of these cases revealed that both giant epidermoid cysts (and the pilonidal cyst in the first case) were located in the inferior part of the pelvis, anterior to the coccyx. This gave us the chance for appropriate mapping of surgery, and the trans-sacral approach for both lesions was considered. Complete surgical removal of the cyst is also the preferred treatment for pilonidal disease. Preoperative misdiagnosis of the epidermoid cyst in this patient could have caused catastrophic peroperative problems such as rectal injury, severe hemorrhage, and vascular injury.

In these cysts, which have similar radiologic findings, definitive diagnosis is generally made by histopathology. The epidermoid cyst has a thin wall lined by keratinised squamous epithelium, without skin adnexa or any muscle. The cysts are filled with desquamated debris and keratin. ${ }^{2,9}$ The first case was diagnosed as an epidermoid cyst, and was lined by keratinised squamous epithelium without skin appendages. The second case was diagnosed as a tail-gut cyst, which was lined by squamous epithelium with appendages and calcified-fibrous areas.

In conclusion, pelvic cystic lessions are extremely rare. Developmental cysts must be kept in mind when large, thin-walled, clear liquid-containing retrorectal-pelvic cysts are seen. Due to malignancy potential, they must be removed entirely, taking care to prevent injury to the anal sphincter and rectum. Preoperative appropriate mapping could give a chance for the trans-sacral approach, thus avoiding intraabdominal complications.

\section{PATIENTS' CONSENT:}

Informed consent was obtained from the patient to publish the data regarding this case.

\section{CONFLICT OF INTEREST:}

Authors declared no conflict of interest.

\section{AUTHORS' CONTRIBUTION:}

KRS: Conception and design, acquisition of data, drafting of manuscript, and critical revision of the manuscript.

$\mathrm{OK}, \mathrm{NAH}$ : Conception and design, drafting of manuscript.

MCT: Acquisition of data, critical revision of the manuscript, supervision.

\section{REFERENCES}

1. Dahan H, Arrivé L, Wendum D. Retrorectal developmental cysts in adults clinical and radiologic-histopathologic review differential diagnosis and treatment. Radiographics 2001; 21 : 575-84.

2. Azatçam M, Altun E, Avci V. Histopathological diagnostic dilemma in retrorectal developmental cysts report of a case and review of the literature. Turk Patoloji Derg 2018; 34: 175-8.

3. Kesici U, Sakman G, Mataraci E. Retrorectal presacralepidermoid cyst report of a case. Eurasian J Med 2013; 45:207-10. 
4. Jain P, Pal DK. Pelvic epidermoid cyst a rare cause of lower urinary tract symptoms. BMJ Case Rep 2018; 2018 pii:bcr2017-223258.

5. Riojas CM, Hahn CD, Johnson EK. Presacral epidermoid cyst in a male a case report and literature review. J Surg Educ 2010; 67:227-32.

6. Hove MG, Gil JM, Guijarro JJ. Laparoscopic approach to tailgut cyst (retrorectal cystic hamartoma). J Minim Access Surg
2019; 15:262-4.

7. Patel N, Maturen KE, Wasnik AP. Imaging of presacral masses a multidisciplinary approach. Brj Radiol 2016; 89:1061.

8. Shetty AS, Loch R, Yoo N, Mellnick V, Fowler K, Narra V. Imaging of tailgut cysts. Abdom Imaging 2015; 40:2783-95.

9. Yang DM, Kim HC, Lee HL, Lee SH, Kim GY. Squamous cell carcinoma arising from a presacral epidermoid cyst CT and MR findings. Abdom Imaging 2008; 33:498-500. 